\title{
Differential Bandpass Filter With Common-Mode Suppression Based on Open Split Ring Resonators and Open Complementary Split Ring Resonators
}

\author{
Paris Vélez, Student Member, IEEE, Jordi Naqui, Student Member, IEEE, \\ A. Fernández-Prieto, Student Member, IEEE, Miguel Durán-Sindreu, Member, IEEE, \\ Jordi Bonache, Member, IEEE, Jesús Martel, Member, IEEE, Francisco Medina, Fellow, IEEE, and \\ Ferran Martín, Fellow, IEEE
}

\begin{abstract}
Differential (balanced) microstrip bandpass filters (BPFs) implemented by combining open split ring resonators (OSRRs) and open complementary split ring resonators (OCSRRs) are proposed. The OSRRs are series connected in both strips of the differential line, whereas the OCSRRs are paired face-to-face and connected between both line strips in a symmetric configuration. For the differential mode, the OCSRRs are virtually connected to ground and the structure can be modeled, to a first-order approximation, by a cascade of series resonators (OSRRs) alternating with shunt resonators (OCSRRs), i.e., the canonical circuit model of a BPF. These filters have the ability to suppress the common mode by properly adjusting the metallic area surrounding the OCSRRs. An order-3 balanced Chebyshev $B P F$ is designed and fabricated to illustrate the possibilities of the approach. The filter does not require vias (contrary to previous single-ended microstrip BPFs based on OSRRs and OCSRRs), filter dimensions are small, and the common mode is efficiently suppressed with more than $20 \mathrm{~dB}$ rejection within the differential filter pass band.
\end{abstract}

Index Terms-Bandpass filter (BPF), differential filter, microstrip, split ring resonators (SRRs).

\section{INTRODUCTION}

$\mathbf{T}$ HE design of differential (balanced) bandpass filters (BPFs) with common-mode noise suppression has been a subject of interest in recent years. Such filters are a demand in modern communication systems based on differential transmission lines and balanced circuits. Several strategies for the implementation of balanced filters with common mode rejection have been proposed, but the interest here is on wideband differential-mode response filters. Thus, balanced BPFs covering the UWB band (3.1 GHz to $10.6 \mathrm{GHz}$ ) have been reported in [1], but filter performance (specially the differential-mode return loss) is poor, and the lack of a common ground plane limits the integration of such balanced filters with other differential-mode

Manuscript received October 11, 2012; accepted December 06, 2012. Date of publication January 01 , 2013; date of current version January 16, 2013. This work was supported by Spain-MICIIN (contracts TEC2010-17512 METATRANSFER and CSD2008-00066) and the Catalan Government through the project 2009SGR-421, and by Grant AP2010-0467.

P. Vélez, J. Naqui, M. Durán-Sindreu, J. Bonache, and F. Martín are with GEMMA/CIMITEC (Departament d'Enginyeria Electrònica), Universitat Autònoma de Barcelona. Barcelona 08193, Spain (e-mail: ferran.martin@uab.es).

A. Fernández-Prieto, J. Martel, and F. Medina are with Grupo de Microondas, Universidad de Sevilla, Sevilla 41012, Spain (e-mail: medina@us.es).

Color versions of one or more of the figures in this paper are available online at http://ieeexplore.ieee.org.

Digital Object Identifier 10.1109/LMWC.2012.2236083 active circuits. A wideband balanced BPF with significant common mode suppression in the differential pass band, and based on a modified two-stage branch line structure with a pair of symmetrically etched open-circuited stubs, has been proposed in [2]. Essentially, the structure behaves as a BPF with quarter wavelength shunt-shorted stubs for the differential mode, whereas the common mode is efficiently suppressed since the stubs are opened for this mode (the rejection level for the common mode is controlled by the pair of loading open-circuited stubs). The filter exhibits good common mode rejection (higher than $20 \mathrm{~dB}$ ) over the differential-mode pass band. However, the filter is essentially a balanced distributed filter based on quarter wavelength transmission line sections and stubs, and hence electrically large (similar wideband balanced filters are reported in [3], [4]).

To alleviate the previous aspect (i.e., filter size), we propose in this letter a novel approach for the design of common-mode suppressed wideband balanced BPFs based on electrically small open resonators, that is, open split ring resonators (OSRRs) [5] and open complementary split ring resonators (OCSRRs) [6]. OSRR- and OCSRR-based single-ended BPFs in coplanar waveguide $(\mathrm{CPW})$ and microstrip technology were already reported [7], [8]. Since OSRRs and OCSRRs are intrinsically broadband resonators, wide filter pass bands can be achieved. The main originality of this paper is to present and discuss the specific arrangement of the elements (OSRRs and OCSRRs) to obtain a differential-mode BPF in microstrip technology, and the strategy to achieve an efficient suppression of the common mode in the differential filter pass band. The proposed lumped element equivalent circuit model will be a helpful tool for design purposes.

\section{DifFerential-Mode BPF BASED ON OSRRS AND OCSRRS: MODELING AND DESIGN}

The single-ended BPFs based on OSRRs and OCSRRs consist on a cascade of series connected OSRRs alternating with shunt connected OCSRRs [7] (Fig. 1(a) depicts a typical topology in microstrip technology). Since the OSRR and the OCSRR are described (to a first-order approximation) by means of a series and a parallel resonant tank, respectively, it follows that the structure synthesizes the canonical circuit model of a BPF. To effectively ground the OCSRRs, it is necessary to include metallic vias in the design, and the ground plane is windowed in the regions beneath the OSRRs in order to obtain 


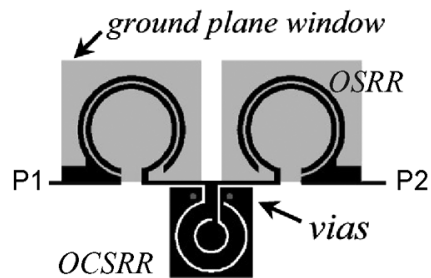

(a)

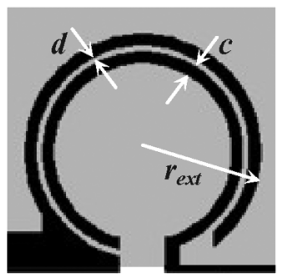

(b)

Fig. 1. Typical topology of a single-ended microstrip T-network order-3 BPF implemented by means of OSRRs and OCSRRs (a), and detail of the OSRR (b), including relevant dimensions. The upper metal level is indicated in black, whereas the ground plane windows are depicted in grey. For the OCSRR, $c$ is the width of the slotted rings, and $d$ their separation.

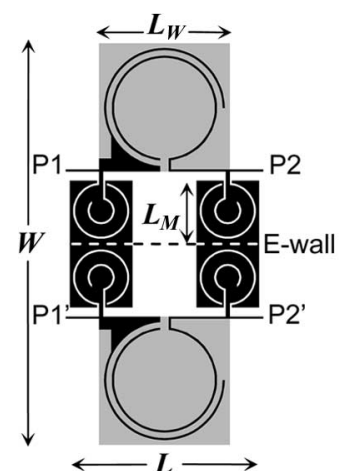

(a)

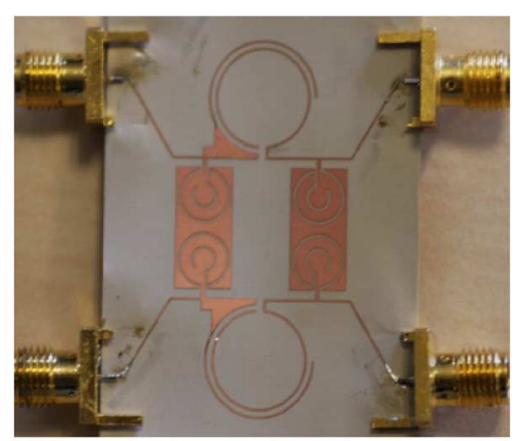

(b)

Fig. 2. Typical layout of the proposed OSRR/OCSRR based differential-mode BPF ( $\pi$-network, order-3) with common mode suppression (a) and fabricated prototype (b). The considered substrate is the Rogers RO3010 with dielectric constant $\varepsilon_{r}=10.2$ and thickness $h=0.254 \mathrm{~mm}$. Dimensions are: $L=$ $18.9 \mathrm{~mm}, W=37.8 \mathrm{~mm}, L_{W}=12.6 \mathrm{~mm}, L_{M}=6 \mathrm{~mm}$. For the OCSRR: $r_{e x t}=2.7 \mathrm{~mm}, c=0.2 \mathrm{~mm}$ and $d=1.2 \mathrm{~mm}$. For the OSRR: $r_{e x t}=$ $5.8 \mathrm{~mm}, c=0.2 \mathrm{~mm}$ and $d=0.55 \mathrm{~mm}$. The $50 \Omega$ microstrip lines have a width of $0.21 \mathrm{~mm}$.

a more accurate description of the particle by means of a series resonator [5], [7].

A typical layout of the proposed differential BPF is depicted in Fig. 2(a). The structure is symmetric with respect to the indicated plane (dashed line). For the differential mode, where there is a virtual ground in that plane (electric wall), the OCSRRs are grounded (without the presence of vias), and the structure exhibits a pass band. If the distance between the host lines is large, no coupling effects take place between mirror elements, and filter design is as simple as designing a single ended BPF.

Several strategies to suppress the common mode in differential lines have been reported in the literature [9]-[11]. Among them, defected-ground structures (DGS), implemented by means of dumbbell shaped resonators [9], U- and H-shaped resonators [10], or complementary split ring resonators (CSRRs) [11], have been demonstrated to be very efficient stop band filters. However, rather than cascading such resonators to the balanced filter, the proposed strategy to suppress the common mode in the region of interest (i.e., the differential filter pass band) consists on tailoring the metallic region surrounding the OCSRRs. This represents an advantage over other proposals [9]-[11] since there is no need of introducing additional elements to the filter (size is saved).

To gain insight on filter design, let us consider the lumped element equivalent circuit model of the four-port section corresponding to one pair of face-to-face OCSRRs (Fig. 3(a)). $L_{p}$ and $C_{p}$ model the OCSRR, $L$ accounts for the inductive strip present between the microstrip lines and the center part of the OCSRRs,

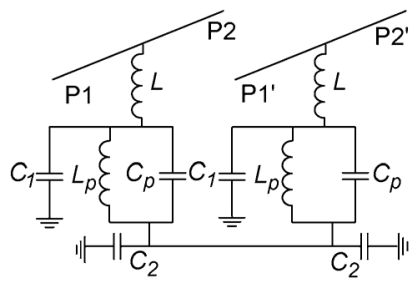

(a)

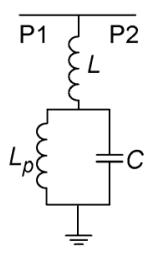

(b)

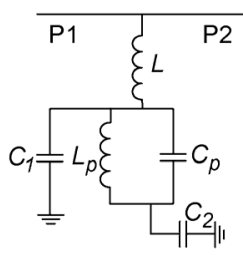

(c)
Fig. 3. Equivalent circuit model of the face-to-face OCSRR pair section. (a) complete model; (b) differential-mode model; (c) common-mode model.

$C_{1}$ is the capacitance between the central strip of the OCSRR and the ground plane; finally, $C_{2}$ is the patch capacitance corresponding to the metallic region surrounding the OCSRRs. The models for the differential and common modes are depicted in Figs. 3(b) and 3(c), respectively. Notice that the capacitance $C_{2}$ is grounded for the differential mode and does not play any role. Thus, for this mode, the shunt OCSRR is described by a parallel resonator in series with an inductor (this inductor is useful to introduce a transmission zero above the differential filter pass band) [8]. However, for the common mode, the symmetry plane is an open-circuit, and the effect of the capacitance $C_{2}$ is the presence of two transmission zeroes in the common mode frequency response. Once the elements $L, L_{p}, C_{p}$ and $C_{1}$ are set to satisfy the differential mode filter response (including the transmission zero above the pass band), $C_{2}$ is adjusted to set the first transmission zero of the common mode frequency response in the centre of the differential mode pass band.

The specifications of the designed differential BPF are: order $n=3$, Chebyshev response with fractional bandwidth $F B W=$ $45 \%$, central frequency $f_{o}=1 \mathrm{GHz}$, and $0.05 \mathrm{~dB}$ ripple. From these specifications, the elements of the canonical $\pi$-network order-3 BPF can be obtained through well known transformations from the low pass filter prototype. Once these elements are known, the topology of the series-connected OSRRs has been obtained by curve fitting the response of the series LC resonator giving the ideal Chebyshev response in the region of interest. For the OCSRRs, the elements of the model of Fig. 3(b) ( $L, L_{p}$, and $C=C_{1}+C_{p}$ ) are derived from the susceptance slope at $f_{o}$ (i.e., by forcing it to be equal to that of the $\mathrm{LC}$ tank giving the ideal Chebyshev response), and from the differential mode transmission zero, given by

$$
f_{Z}^{d d}=\frac{1}{2 \pi} \sqrt{\frac{1}{C}\left(\frac{1}{L}+\frac{1}{L_{p}}\right)}
$$

The parasitic capacitances of the $\pi$-model of the OSRR (which can be easily extracted from the electromagnetic simulation of the isolated particle) [7] are small and have negligible effect on the differential filter response. Once $L, L_{p}$, and $C$ are known, the OCSRRs are synthesized with the help of the model reported in [6] and a parameter extraction procedure similar to that reported in [8]. From this model, the OCSRR capacitance, $C_{p}$, can be estimated and hence $C_{1}$ can be derived. Finally, $C_{2}$ is adjusted to the required value to force the common mode transmission zero at $f_{o}$. The metallic region surrounding the OCSRRs is then expanded or contracted to adjust the common mode transmission zero to that value (the initial size is inferred from the parallel plate capacitor formula). Following this procedure, the element values and the layout of the shunt branch have been inferred. The differential and common mode frequency response 

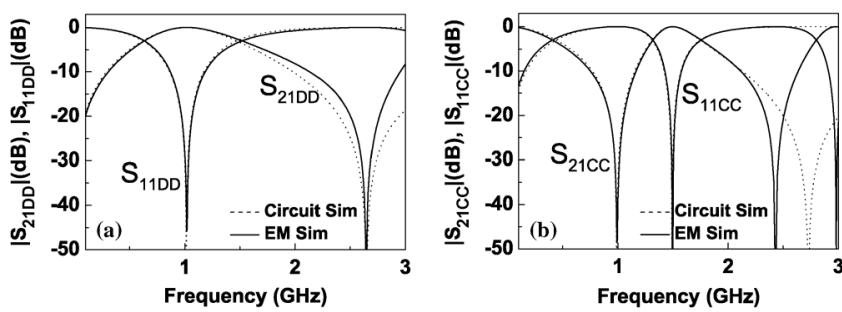

Fig. 4. Circuit and electromagnetic simulation of the differential (a) and common (b) mode frequency response of the OCSRR pair section. Element values are: $L=0.628 \mathrm{nH}, L_{p}=3.675 \mathrm{nH}, C_{p}=0.1 \mathrm{pF}, C_{1}=6.85 \mathrm{pF}$, $C_{2}=5.65 \mathrm{pF}$.

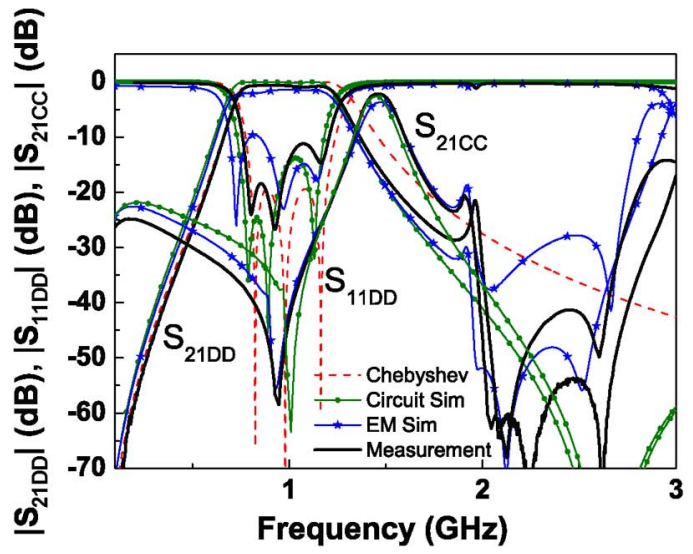

Fig. 5. Simulated and measured differential and common mode insertion loss, and differential mode return loss. The circuit simulations and the Chebyshev response are included. For the $\pi$-model of the OSRR, the elements of the series LC tank are $22.28 \mathrm{nH}$ and $1.22 \mathrm{pF}$, and the values of the shunt capacitors are $1.35 \mathrm{pF}$ (left) and $0.74 \mathrm{pF}$ (right). The measured differential insertion and return loss verify IL $<1.2 \mathrm{~dB}$ and $\mathrm{RL}>11 \mathrm{~dB}$ within the interval $0.76 \mathrm{GHz}-1.20$ GHz. Notice that the common mode is rejected with more than $20 \mathrm{~dB}$ at DC.

TABLE I

COMPARISON OF VARIOUS DIFFERENTIAL BANDPASS FILTERS

\begin{tabular}{c|c|c|c|c|c|c}
\hline Ref. & $\boldsymbol{F B W}$ & $\boldsymbol{n}$ & $\begin{array}{c}\boldsymbol{C M R R} \\
\boldsymbol{a} \boldsymbol{f}_{o}\end{array}$ & $f_{\text {Icc }} / f_{\text {Idd }}$ & $f_{2 c c} / f_{2 d d}$ & $\begin{array}{c}\text { Electrical } \\
\text { Size }\end{array}$ \\
\hline$[2]$ & $65 \%$ & 3 & $29 \mathrm{~dB}$ & 0.96 & 1.09 & $0.5 \lambda_{\mathrm{g}} \times 0.5 \lambda_{g}$ \\
{$[3]$} & $50 \%$ & 3 & $48 \mathrm{~dB}$ & 1.25 & 0.94 & $0.5 \lambda_{\mathrm{g}} \times 0.5 \lambda_{g}$ \\
{$[4]$} & $55 \%$ & 3 & $48 \mathrm{~dB}$ & 0.93 & 1.08 & $0.5 \lambda_{\mathrm{g}} \times 0.5 \lambda_{g}$ \\
This work & $\mathbf{4 5 \%}$ & $\mathbf{3}$ & $\mathbf{5 3} \mathrm{dB}$ & $\mathbf{0}$ & $\mathbf{1 . 0 4}$ & $\mathbf{0 . 1 5} \lambda_{\mathrm{g}} \times \mathbf{0 . 3} \lambda_{g}$ \\
\hline
\end{tabular}

of the OCSRR section is shown in Fig. 4. The good agreement between circuit and electromagnetic simulations (inferred from Agilent $A D S$ ) in the region of interest is appreciable and validates the proposed model.

\section{RESULTS}

The photograph of the fabricated filter is shown in Fig. 2(b) (dimensions are $0.15 \lambda_{g} \times 0.30 \lambda_{g}, \lambda_{g}$ being the guided wavelength at the central filter frequency). Fig. 5 shows the simulated and measured insertion and return loss for the differential mode, as well as the insertion loss for the common mode. Experimental data have been obtained by means of the 4-port Agilent PNA $N 5221 A$ network analyzer. We have also included in the figure the circuit simulation for the differential and common mode models. Concerning the $\pi$-network model of the OSRR (shunt capacitances and a series LC tank [7]), the elements are indicated in the caption of Fig. 5. Both the differential and common mode filter responses are in good agreement with the circuit simulations up to roughly $2 \mathrm{GHz}$. The agreement with the Cheby- shev response is also good, although the selectivity and stopband rejection above the pass band of the proposed balanced filter are better due to the effects of the transmission zero (the first spurious appears at roughly $3 f_{o}$ ). As expected, there is a transmission zero at $f_{o}$ for the common mode, and the common mode rejection within the differential filter pass band is better than $20 \mathrm{~dB}$.

A comparison with other wideband balanced filters is given in Table I. The common mode rejection ratio $(C M R R)$ is the ratio between $\left|S_{21}\right|$ for the common mode and the differential mode, expressed in $\mathrm{dB}$. In the table, $f_{1 d d}$ and $f_{2 d d}$ are the lower and upper differential mode cutoff frequencies, respectively, and $f_{1 c c}$ and $f_{2 c c}$ are the $-20 \mathrm{~dB}$ common mode cutoff frequencies. In view of the table, filter size, $C M R R$, and common-mode rejection bandwidth (at $-20 \mathrm{~dB}$ ) relative to the differential-mode bandwidth, are found to be competitive.

\section{CONCLUSION}

It has been demonstrated that balanced BPFs with inherent common mode rejection can be realized by combining OSRRs and OCSRRs. Easy design and fabrication, small size, broad pass bands, and high common-mode rejection within the differential filter pass band are the main advantages.

\section{REFERENCES}

[1] A. Saitou, K. P. Ahn, H. Aoki, K. Honjo, and K. Watanabe, "Differential-mode bandpass filters with four coupled lines embedded in self complementary antennas," IEICE Trans. Electron., vol. E90-C, no. 7, pp. 1524-1532, Jul. 2007.

[2] T. B. Lim and L. Zhu, "A differential-mode wideband bandpass filter on microstrip line for UWB applications," IEEE Microw. Wireless Compon. Lett., vol. 19, no. 10, pp. 632-634, Oct. 2009.

[3] T. B. Lim and L. Zhu, "Highly selective differential-mode wideband bandpass filter for UWB application," IEEE Microw. Wireless Compon. Lett., vol. 21, no. 3, pp. 133-135, Mar. 2011.

[4] M. Li, F. Zhang, K. Choi, J. Lee, and J. C. Lee, "A differential-mode wideband band-pass filter for UWB application," in Proc. PIERS'12, Kuala Lumpur, Malaysia, Mar. 2012, pp. 1237-1240.

[5] J. Martel, R. Marqués, F. Falcone, J. D. Baena, F. Medina, F. Martín, and M. Sorolla, "A new LC series element for compact band pass filter design," IEEE Microw. Wireless Comp. Lett., vol. 14, no. 5, pp. 210-212, May 2004.

[6] A. Velez, F. Aznar, J. Bonache, M. C. Velázquez-Ahumada, J. Martel, and F. Martín, "Open complementary split ring resonators (OCSRRs) and their application to wideband CPW band pass filters," IEEE Microw. Wireless Comp. Lett., vol. 19, no. 4, pp. 197-199, Apr. 2009.

[7] M. Durán-Sindreu, A. Vélez, F. Aznar, G. Sisó, J. Bonache, and F. Martín, "Application of open split ring resonators and open complementary split ring resonators to the synthesis of artificial transmission lines and microwave passive components," IEEE Trans. Microw. Theory Tech., vol. 57, no. 12, pp. 3395-3403, Dec. 2009.

[8] M. Durán-Sindreu, P. Vélez, J. Bonache, and F. Martín, "Broadband microwave filters based on Open Split Ring Resonators (OSRRs) and Open Complementary Split Ring Resonators (OCSRRs): Improved models and design optimization," Radioengineering, vol. 20, pp. 775-783, Dec. 2011.

[9] W. T. Liu, C.-H. Tsai, T.-W. Han, and T.-L. Wu, "An embedded common-mode suppression filter for $\mathrm{GHz}$ differential signals using periodic defected ground plane," IEEE Microw. Wireless Comp. Lett., vol. 18, no. 4, pp. 248-250, Apr. 2008.

[10] S.-J. Wu, C.-H. Tsai, T.-L. Wu, and T. Itoh, "A novel wideband common-mode suppression filter for gigahertz differential signals using coupled patterned ground structure," IEEE Trans. Microw. Theory Techn., vol. 57, no. 4, pp. 848-855, Apr. 2009.

[11] J. Naqui, A. Fernández-Prieto, M. Durán-Sindreu, J. Selga, F. Medina, F. Mesa, and F. Martín, "Split rings-based differential transmission lines with common-mode suppression," in IEEE MTT-S Int. Dig., Baltimore, MD, Jun. 2011. 